(C) The Author, 2014. Journal compilation (C) Australian Museum, Sydney, 2014

Technical Reports of the Australian Museum, Online (2014) No. 24, pp. 61-63.

ISSN 1835-4211 (online)

http://dx.doi.org/10.3853/j.1835-4211.24.2014.1618

\title{
Establishing Priorities for Research on the Epidemiology of Koala Retrovirus (KoRV) in Koalas (Phascolarctos cinereus)
}

\author{
Carmel L. Witte \\ Wildlife Diseases Laboratories, Institute for Conservation Research, \\ San Diego Zoo Global, San Diego, CA 92101, United States of America \\ cwitte@sandiegozoo.org
}

\begin{abstract}
This manuscript summarizes the break-out session held on the epidemiology of disease expression of koala retrovirus (KoRV) in koalas (Phascolarctos cinereus) at the Koala Conservation Workshop: The koala and its retroviruses: implications for sustainability and survival held at San Diego Zoo, April 17-18, 2013. The goals of this break-out session were to develop and prioritize specific research goals related to KoRV epidemiology, to identify actions, and to determine the responsible parties and timelines. Identified areas for epidemiologic research include studies in both wild and captive populations. For wild populations, baseline estimates of incidence and prevalence that account for potential biases in surveillance are needed. Landscape-level studies that determine whether KoRV contributes to the decline or stability of wild populations are also a priority. Captive populations with high-quality health data and management records can provide opportunities to identify factors associated with disease expression. These populations may also be pivotal in understanding the clinical importance of different KoRV subtypes.
\end{abstract}

WitTe, CARMEL L. 2014. Establishing priorities for research on the epidemiology of koala retrovirus (KoRV) in koalas (Phascolarctos cinereus). In The Koala and its Retroviruses: Implications for Sustainability and Survival, ed. Geoffrey W. Pye, Rebecca N. Johnson and Alex D. Greenwood. Technical Reports of the Australian Museum, Online 24: 61-63.

Relevant to any epidemiologic study are the two important questions: What do we already know and what are the current gaps in knowledge?

What do we know? There are koala populations with low KoRV prevalence and with no disease expression. The Kangaroo Island population is a good example of lower KoRV prevalence with little disease expression (Simmons et al., 2012). There are also populations with high KoRV prevalence with little disease expression (e.g., St. Bees Island) (Tarlinton et al., 2006; Bill Ellis, pers. comm. 2014). There appears to be a difference in prevalence and disease expression between populations of northern koalas versus southern koalas (Simmons et al., 2012). There also appears to be similar prevalence of KoRV with widely differing prevalence of disease expression between southeastern and central Queensland koala populations (Simmons et al., 2013; Amber Gillett and Sean FitzGibbon pers. comm. 2013). One challenge in Australia is that there have only been a few studies where sampling and testing was limited and opportunistic.

Gaps in knowledge. Currently the important gaps in knowledge are: Does KoRV causes disease in koalas and if so, is it associated with declines? What diseases (e.g., neoplasia) are caused by KoRV in koalas? Are there environmental, social, or other triggers for disease expression? What role do the exogenous and endogenous variants of KoRV play in causing disease? Does KoRV viral load increase with age? 